\title{
Raman optical activity spectra from density functional perturbation theory and density-functional-theory-based molecular dynamics
}

\author{
Luber, Sandra
}

\begin{abstract}
We describe the calculation of Raman optical activity (ROA) tensors from density functional perturbation theory, which has been implemented into the CP2K software package. Using the mixed Gaussian and plane waves method, ROA spectra are evaluated in the double-harmonic approximation. Moreover, an approach for the calculation of ROA spectra by means of density functional theory-based molecular dynamics is derived and used to obtain an ROA spectrum via time correlation functions, which paves the way for the calculation of ROA spectra taking into account anharmonicities and dynamic effects at ambient conditions.
\end{abstract}

DOI: https://doi.org/10.1021/acs.jctc.6b00820

Posted at the Zurich Open Repository and Archive, University of Zurich ZORA URL: https://doi.org/10.5167/uzh-136559

Journal Article

Accepted Version

Originally published at:

Luber, Sandra (2017). Raman optical activity spectra from density functional perturbation theory and density-functional-theory-based molecular dynamics. Journal of Chemical Theory and Computation, 13(3):1254-1262.

DOI: https://doi.org/10.1021/acs.jctc.6b00820 


\title{
Raman optical activity spectra from density functional perturbation theory and density functional theory-based molecular dynamics
}

\author{
Sandra Luber ${ }^{1}$ \\ Department of Chemistry C, University of Zurich, Winterthurerstrasse 190, \\ 8057 Zurich, Switzerland
}

\footnotetext{
${ }^{1}$ E-Mail: sandra.luber@chem.uzh.ch
} 


\begin{abstract}
We describe the calculation of Raman optical activity (ROA) tensors from density functional perturbation theory, which has been implemented into the $\mathrm{CP} 2 \mathrm{~K}$ software package. Using the mixed Gaussian and plane waves method, ROA spectra are evaluated in the double-harmonic approximation. Moreover, an approach for the calculation of ROA spectra by means of density functional theory-based molecular dynamics is derived and used to obtain an ROA spectrum via time correlation functions, which paves the way for the calculation of ROA spectra taking into account anharmonicities and dynamic effects at ambient conditions.
\end{abstract}




\section{Introduction}

Vibrational spectroscopy is an important method for the elucidation of structure and dynamics of compounds used in chemistry, biology, physics, and materials and environmental sciences. Among the different techniques developed, Infrared (IR) and Raman spectroscopy are the most common ones and standard approaches for the investigation of chemical systems. The measured spectra can be easily analyzed for structurally simple, small molecules but become increasingly crowded and complicated for larger compounds. This is even more true for the chiral variants of IR and Raman spectroscopy, vibrational circular dichroism $(\mathrm{VCD})^{1,2}$ and vibrational Raman optical activity $(\mathrm{ROA})^{3-6}$ spectroscopy, respectively, which are sensitive to the chirality of the compounds under study leading to spectra featuring in general positive and negative band intensities for chiral systems. Whereas VCD is nowadays a well established method, ROA spectroscopy is less widespread and has been mostly used for biomolecules in solution (for a recent review, see Ref. $\left.{ }^{7}\right)$.

Recent advances in ROA measurements include the development of a spectrometer in the deep-ultraviolet region ${ }^{8}$ and observation of paramagnetic ROA. ${ }^{9}$ Calculations have profited from various developments such as analytical ROA intensity differences, ${ }^{10}$ efficient density fitting for ROA property tensors in combination with a massive parallel environment, ${ }^{11}$ Cartesian transfer ${ }^{12}$ and molecules-in-molecules fragment ${ }^{13}$ techniques, $^{2}$ and coupled cluster ${ }^{14}$ and intensity-tracking ${ }^{15-17}$ approaches. ROA computations have been presented for various biomolecules ranging from carbohydrates ${ }^{17-19}$ up to complex proteins $^{20-27}$ (for reviews, see Refs. ${ }^{28-30}$ ) as well as polymers. ${ }^{31,32}$ In contrast to that, calculation of Resonance ROA is a rather unexplored, yet promising field, ${ }^{33-36}$ in particular for metal complexes. ${ }^{35,37}$ Simulated ROA spectra of the latter are in general still quite rare $^{11,27,35,38,39}$ and we refer to Ref. ${ }^{37}$ for a recent review about ROA of transition metalcontaining coordination compounds and solids. In order to be able to interpret the spectra 
and to obtain in-depth knowledge about the structure and properties of the molecules, calculations are indispensable and various analysis tools have been developed. ${ }^{20,40-42}$

The harmonic approximation, in combination with Kohn-Sham (KS) density functional theory (DFT), is currently the standard way to obtain vibrational spectra leading to a modest computational cost. This comes with several drawbacks since no dynamics and anharmonicities are included into the calculation and the system under study is assumed to be at its equilibrium structure based on a temperature of $0 \mathrm{~K}$, which can be especially problematic for molecules with conformational degrees of freedom and hydrogen-bonded systems. ${ }^{43}$ Since such calculations directly provide solely vibrational frequencies and corresponding intensities (depending on the type of spectrum computed), the band shapes are obtained a posteriori by applying an artificial broadening. Anharmonic effects may be included (see, for instance, Ref. ${ }^{44}$ for a review) and several examples for ROA have been presented in Refs. ${ }^{45,46}$ as well as recently in Ref. ${ }^{47}$ building upon the implementation described in Ref. ${ }^{11}$

An alternative way for the calculation of vibrational spectra is based on DFT-based molecular dynamics (MD) where the conformational phase space, hydrogen-bonding dynamics, and other local geometry arrangements can be explored at ambient conditions, taking into account realistic thermodynamic conditions as employed in experiment. ${ }^{48}$ Vibrational spectra can be obtained from DFT-based MD via Fourier transformation of certain time correlation functions. This ansatz has the advantage that band shapes are directly obtained from the calculation and no artificial broadening has to be applied. ${ }^{49,50}$ Moreover, anharmonicity effects are included since no specific shape of the potential energy surface is assumed in contrast to the harmonic approximation. ${ }^{49,50}$ The power spectrum, for example, contains bands at all vibrational frequencies and can be calculated from the time autocorrelation function of the nuclear velocities. Examples include IR spectra, which are obtained from time autocorrelation functions of the electric dipole moment 
and have often been reported in the literature (see, e.g., Refs. ${ }^{49,50}$ and references cited therein). Sum frequency generation spectra ${ }^{51-56}$ and Raman spectra from DFT-based MD have been rarely reported. ${ }^{50,57-60}$ Very recently, VCD spectra obtained from DFT-based MD have been presented as well. ${ }^{61}$ In the framework of ROA spectroscopy, (classical or DFT-based) MD has solely been employed to sample solute-solvent clusters ${ }^{19,39,62-67}$ and a ionic liquid, ${ }^{68}$ in this way providing input model structures for static calculations. Whereas classical trajectories have been used to calculate ROA spectra of model harmonic systems, ${ }^{69}$ no ROA spectrum directly evaluated from DFT-based MD has been presented to the best of our knowledge.

In this manuscript, we describe the calculation of ROA spectra using the mixed Gaussian and plane waves approach. ${ }^{70}$ The ROA polarizability tensors are efficiently evaluated by means of density functional perturbation theory (DFPT), which we have recently adapted to the calculation of Raman spectra. ${ }^{60}$ ROA spectra cannot only be obtained based on the standard approach within the double-harmonic approximation ${ }^{71-73}$ but also using time correlation functions obtainable from DFT-based MD. This opens a novel way for the calculation of ROA spectra by considering the complex dynamics of the system under study, thus naturally including finite temperature effects and anharmonicities.

The manuscript is organized as follows: Section 2 provides theoretical basics for the calculation of ROA intensity differences (Section 2.1) and a description of the implementation for the evaluation of ROA polarizability tensors and achievement of gauge-origin independent results (Section 2.2). After the computational methodology in Section 3, example calculations for $S$-methyloxirane in the static double-harmonic approximation and via time correlation function from DFT-based MD can be found in Section 4. A summary and outlook is given in Section 5 . 


\section{Theoretical background}

\subsection{Raman optical activity intensity differences}

Employing Placzek's polarizability theory ${ }^{74}$ the ROA intensity difference for the backscattering experimental set-up in the far from resonance approximation (i.e. the incident light is far away from any resonance with an electronic transition) is given by ${ }^{72,73}$

$$
\left(I_{R}-I_{L}\right)\left(180^{\circ}\right) \propto \beta\left(\mathbf{G}^{\prime}\right)^{2}+\frac{1}{3} \beta(\mathbf{A})^{2} .
$$

For non-metallic systems and the static limit $\omega_{L} \rightarrow 0\left(\omega_{L}\right.$ is the angular frequency of the incident light), the anisotropic ROA invariants (summation over Greek indices is implied in this subsection) are obtained $\mathrm{as}^{75}$

$$
\begin{gathered}
\beta\left(\mathbf{G}^{\prime}\right)^{2}=\frac{1}{2}\left(3 \alpha_{\alpha \beta} G_{\alpha \beta}^{\prime s l}-\alpha_{\alpha \alpha} G_{\beta \beta}^{\prime s l}\right) \\
\beta(\mathbf{A})^{2}=\frac{1}{2} \alpha_{\alpha \beta} \epsilon_{\alpha \gamma \delta} A_{\gamma, \delta \beta} .
\end{gathered}
$$

$\alpha_{\alpha \beta}$ and $G_{\alpha \beta}^{\prime s l}$ are the $\alpha \beta$ components (the Greek subscripts $\alpha$ and $\beta$ represent the spatial x-, y-, and z-coordinates) of the electric-dipole-electric-dipole tensor $\boldsymbol{\alpha}$ and the electricdipole-magnetic-dipole tensor $G^{\prime s l}$ (the superscript "sl" indicates the static limit), respectively. $A_{\gamma, \delta \beta}$ is the $\gamma \delta \beta$ component of the electric-dipole-electric-quadrupole polarizability tensor $\boldsymbol{A}, \epsilon_{\alpha \gamma \delta}$ the $\alpha \gamma \delta$ component of the (Levi-Civita) third-rank antisymmetric unit tensor. In the double-harmonic approximation, ${ }^{71}$ the ROA intensity differences are obtained by calculation of the derivative of the ROA tensor components with respect to normal coordinates. In MD, the ROA intensity can be evaluated by Fourier transform of the time crosscorrelation function of the ROA tensor elements,

$$
\begin{aligned}
\left(\beta\left(\mathbf{G}^{\prime}\right)^{2}\right)_{\mathrm{MD}}= & \frac{1}{2} \frac{1}{2 \pi}\left\{\int_{-\infty}^{\infty} \mathrm{d} t \exp \{-\mathrm{i} \omega t\}\left\langle 3 \alpha_{\alpha \beta}(0) G_{\alpha \beta}^{\prime s l}(t)\right\rangle\right. \\
& \left.-\int_{-\infty}^{\infty} \mathrm{d} t \exp \{-\mathrm{i} \omega t\}\left\langle\alpha_{\alpha \alpha}(0) G_{\beta \beta}^{\prime s l}(t)\right\rangle\right\} \\
\left(\beta(\mathbf{A})^{2}\right)_{\mathrm{MD}}= & \frac{1}{2} \frac{1}{2 \pi} \int_{-\infty}^{\infty} \mathrm{d} t \exp \{-\mathrm{i} \omega t\} \epsilon_{\alpha \gamma \delta}\left\langle\alpha_{\alpha \beta}(0) A_{\gamma, \delta \beta}(t)\right\rangle
\end{aligned}
$$


where we used the classical limit of the Kubo transformed time correlation function in analogy to Ref. ${ }^{43}$

\subsection{Evaluation of Raman optical activity tensors}

For nonperiodic systems, the $\boldsymbol{\alpha}$ and $\boldsymbol{A}$ tensor components are evaluated according to ${ }^{72}$

$$
\begin{aligned}
\alpha_{\alpha \beta} & =f_{\text {occ }} \frac{2}{\hbar} \sum_{i, k} \frac{\left\langle\psi_{i}^{(0)}\left|d_{\alpha}\right| \psi_{k}^{(e)}\right\rangle\left\langle\psi_{k}^{(e)}\left|d_{\beta}\right| \psi_{i}^{(0)}\right\rangle}{\omega_{k, e}-\omega_{i, 0}} \\
A_{\alpha, \beta \gamma} & =f_{\text {occ }} \frac{2}{\hbar} \sum_{i, k} \frac{\left\langle\psi_{i}^{(0)}\left|d_{\alpha}\right| \psi_{k}^{(e)}\right\rangle\left\langle\psi_{k}^{(e)}\left|\theta_{\beta \gamma}\right| \psi_{i}^{(0)}\right\rangle}{\omega_{k, e}-\omega_{i, 0}} .
\end{aligned}
$$

$\hbar$ is Planck's constant divided by $2 \pi,\left(\omega_{k, e}-\omega_{i, 0}\right)$ is the angular frequency difference corresponding to the KS orbitals $\psi_{k}^{(e)}$ and $\psi_{i}^{(0)}$ of electronically excited state (indicated via the superscript "(e)") and electronic ground state (labeled by the superscript "(0)"), respectively. The occupation number $f_{\text {occ }}$ has values of 1 and 2 in the unrestricted and closed-shell restricted KS approach, respectively. $d_{\alpha}=-e r_{\alpha}=-e \sum_{l=1}^{N} r_{\alpha, l}$ is the $\alpha$ component of the electric dipole operator for the electrons in the length representation, $N$ the number of electrons, $e$ the elementary charge, and $r_{\alpha, l}$ the $\alpha$ component of the position operator of electron $l . \boldsymbol{\theta}$ is the electronic part of the electric quadrupole operator in its traceless form with components

$$
\theta_{\alpha \beta}=-\frac{1}{2} e \sum_{l=1}^{N}\left(3 r_{\alpha, l} r_{\beta, l}-\boldsymbol{r}_{l}^{2} \delta_{\alpha \beta}\right) .
$$

The $\boldsymbol{G}^{\prime s l}$ tensor components are given as ${ }^{76}$

$$
G_{\alpha \beta}^{\prime s l}=\left(\frac{1}{\omega_{L}} G_{\alpha \beta}^{\prime}\right)_{\omega_{L}=0}=-f_{\mathrm{occ}} \operatorname{Im} \frac{2}{\hbar} \sum_{i, k} \frac{\left\langle\psi_{i}^{(0)}\left|d_{\alpha}\right| \psi_{k}^{(e)}\right\rangle\left\langle\psi_{k}^{(e)}\left|m_{\beta}\right| \psi_{i}^{(0)}\right\rangle}{\left(\omega_{k, e}-\omega_{i, 0}\right)^{2}}
$$

with the electronic part of the magnetic dipole operator $\left(m_{e}\right.$ is the electron mass)

$$
m_{\alpha}=-\sum_{l=1}^{N} \frac{e}{2 m_{e}} \epsilon_{\alpha \beta \gamma} r_{\beta, l} p_{\gamma, l} .
$$


$\boldsymbol{p}_{l}=-\mathrm{i} \hbar \boldsymbol{\nabla}_{l}$ is the momentum operator and the spin-dependent part of the magnetic dipole operator is neglected. The tensor $\boldsymbol{G}^{\prime}$ vanishes for $\omega_{L}=0$.

The electric-dipole-electric-dipole polarizability $\boldsymbol{\alpha}$ can be evaluated via DFPT 77,78 in the electric-dipole approximation. The external electric field $\boldsymbol{E}$ is considered as a perturbative parameter $\lambda$ coupled to the electronic part of the electric dipole moment leading to a perturbation $\lambda \epsilon^{(1)}=-\sum_{\beta=1}^{3} E_{\beta} f_{\text {occ }} \sum_{i}\left\langle\psi_{i}^{(0)}\left|d_{\beta}\right| \psi_{i}^{(0)}\right\rangle$ added to the unperturbed KS energy functional $\epsilon^{(0)}{ }^{79}$ The total energy functional for the perturbation in $\beta$ direction is given as

$$
\epsilon=\epsilon^{(0)}\left[\left\{\left|\psi_{i}^{(0)}\right\rangle-E_{\beta}\left|\psi_{i, d}^{(1), \beta}\right\rangle+\ldots\right\}\right]-E_{\beta} \epsilon^{(1, \beta)}\left[\left\{\left|\psi_{i}^{(0)}\right\rangle-E_{\beta}\left|\psi_{i, d}^{(1), \beta}\right\rangle+\ldots\right\}\right] .
$$

$\left|\psi_{i}^{(0)}\right\rangle$ and $\left|\psi_{i, d}^{(1), \beta}\right\rangle$ are the unperturbed and first-order perturbed KS orbitals, respectively.

Our implementation for non-hybrid exchange-correlation density functionals is described in the following. The first-order perturbed KS orbitals $\left\{\left|\psi_{j, d}^{(1), \beta}\right\rangle\right\}$ are calculated via the inhomogeneous set of coupled equations (indicating here the dependence on the spatial coordinates explicitly)

$$
\begin{array}{r}
-P \sum_{j}\left(H^{(0)} \delta_{i j}-\left\langle\psi_{i}^{(0)}\left|H^{(0)}\right| \psi_{j}^{(0)}\right\rangle\right) P\left|\psi_{j, d}^{(1), \beta}\right\rangle= \\
P\left[\left.\int \frac{\partial^{2} E_{\mathrm{Hxc}}[n(\boldsymbol{r})]}{\partial n(\boldsymbol{r}) \partial n\left(\boldsymbol{r}^{\prime}\right)}\right|_{n^{(0)}(\boldsymbol{r})} n_{d}^{(1), \beta}\left(\boldsymbol{r}^{\prime}\right) d \boldsymbol{r}^{\prime}\left|\psi_{i}^{(0)}\right\rangle+d_{\beta}\left|\psi_{i}^{(0)}\right\rangle\right]
\end{array}
$$

employing the orthogonality condition

$$
\left\langle\psi_{i}^{(0)} \mid \psi_{j, d}^{(1, \beta)}\right\rangle=0 \quad \forall i, j
$$

and the projector on the manifold of the unoccupied states

$$
P=1-\sum_{i}\left|\psi_{i}^{(0)}\right\rangle\left\langle\psi_{i}^{(0)}\right|
$$

$E_{\mathrm{Hxc}}[n(\boldsymbol{r})]$ is the Hartree, exchange, and correlation energy functional and $H^{(0)}$ is the $\mathrm{KS}$ Hamiltonian of the unperturbed ground state. 
The first-order perturbed density is calculated as

$$
n_{d}^{(1), \beta}(\boldsymbol{r})=\sum_{i} f_{\mathrm{occ}}\left[\psi_{i}^{(0) *}(\boldsymbol{r}) \psi_{i, d}^{(1), \beta}(\boldsymbol{r})+\psi_{i, d}^{(1), \beta *}(\boldsymbol{r}) \psi_{i}^{(0)}(\boldsymbol{r})\right]
$$

The $\alpha \beta$ component of the electric-dipole-electric-dipole polarizability $\boldsymbol{\alpha}$ for nonperiodic systems is obtained as

$$
\alpha_{\alpha \beta}=-2 f_{\text {occ }} \sum_{i}\left\langle\psi_{i, d}^{(1), \alpha}\left|d_{\beta}\right| \psi_{i}^{(0)}\right\rangle
$$

The components of the $\boldsymbol{A}$ tensors can efficiently be computed without an additional DFPT calculation according to

$$
A_{\alpha, \beta \gamma}=-2 f_{\mathrm{occ}} \sum_{i}\left\langle\psi_{i, d}^{(1), \alpha}\left|\theta_{\beta \gamma}\right| \psi_{i}^{(0)}\right\rangle
$$

In order to calculate the $\boldsymbol{G}^{\prime s l}$ tensor, it is necessary to additionally solve the system of linear equations in Eq. (11) with the magnetic field $\boldsymbol{B}$ as the perturbative parameter coupled to the electronic part of the magnetic dipole moment. Since the magnetic dipole operator is imaginary and we use real ground state KS orbitals, the first-order perturbed KS orbitals are imaginary leading to a vanishing first-order perturbed density. This significantly simplifies the system of linear equations in Eq. (11) leading to so-called uncoupled perturbed self-consistent field equations. The resulting first-order perturbed KS orbitals $\left\{\left|\psi_{i, m}^{(1), \beta}\right\rangle\right\}$ are required to calculate $\boldsymbol{G}^{\prime s l}$ :

$$
G_{\alpha \beta}^{\prime s l}=-2 \hbar f_{\mathrm{occ}} \operatorname{Im} \sum_{i}\left\langle\psi_{i, d}^{(1), \alpha} \mid \psi_{i, m}^{(1), \beta}\right\rangle
$$

The ROA invariants are experimentally observable and thus origin independent. In calculations as presented in this work, however, this is not necessarily the case due to the limited size of the basis sets. The electric dipole moment is origin independent if the total charge is zero. ${ }^{73}$ The electric-dipole-electric-dipole polarizability tensor $\boldsymbol{\alpha}$ is also origin 
independent: shifting the origin from $\boldsymbol{O}$ to $\boldsymbol{O}+\boldsymbol{a}$ results in matrix elements

$$
\begin{aligned}
\left\langle\psi_{i}^{(0)}\left|d_{\alpha}(\boldsymbol{O}+\boldsymbol{a})\right| \psi_{k}^{(e)}\right\rangle & =-\left\langle\psi_{i}^{(0)}\left|e \sum_{l=1}^{N}\left[r_{\alpha, l}(\boldsymbol{O})-a_{\alpha, l}\right]\right| \psi_{k}^{(e)}\right\rangle \\
& =\left\langle\psi_{i}^{(0)}\left|d_{\alpha}(\boldsymbol{O})\right| \psi_{k}^{(e)}\right\rangle, \quad \forall \quad\left|\psi_{i}^{(0)}\right\rangle \neq\left|\psi_{k}^{(e)}\right\rangle .
\end{aligned}
$$

The situation is different for the $\boldsymbol{G}^{\prime s l}$ and $\boldsymbol{A}$ tensors, which are gauge origin dependent. ${ }^{73,80}$ The matrix elements containing the electronic part of the magnetic dipole and electric quadrupole operators transform under a shift of the common origin from $\boldsymbol{O}$ to the common origin $\boldsymbol{O}+\boldsymbol{a} \operatorname{as}^{73,80,81}$

$$
\begin{aligned}
&\left\langle\psi_{k}^{(e)}\left|m_{\alpha}(\boldsymbol{O}+\boldsymbol{a})\right| \psi_{i}^{(0)}\right\rangle=-\left\langle\psi_{k}^{(e)}\left|\frac{e}{m_{e}} \sum_{l=1}^{N} \frac{1}{2} \epsilon_{\alpha \beta \gamma}\left(r_{\beta, l}(\boldsymbol{O})-a_{\beta, l}\right) p_{\gamma, l}\right| \psi_{i}^{(0)}\right\rangle \\
&=\left\langle\psi_{k}^{(e)}\left|m_{\alpha}(\boldsymbol{O})\right| \psi_{i}^{(0)}\right\rangle \\
&+\left\langle\psi_{k}^{(e)}\left|\frac{e}{m_{e}} \sum_{l=1}^{N} \frac{1}{2}\left(\boldsymbol{a}_{l} \times \boldsymbol{p}_{l}\right)_{\alpha}\right| \psi_{i}^{(0)}\right\rangle \\
& \forall\left|\psi_{i}^{(0)}\right\rangle \neq\left|\psi_{k}^{(e)}\right\rangle
\end{aligned}
$$

Evaluation of the $\beta(\mathbf{A})^{2}$ invariant in Eq. (3) leads to cancellation of the terms arising from the origin dependence of the $\boldsymbol{A}$ tensor. This is the case for the $\beta\left(\mathbf{G}^{\prime}\right)^{2}$ invariant only if the velocity representation for the electronic part of the electric dipole operator is used in the calculation of the $\boldsymbol{G}^{\prime s l}$ and $\boldsymbol{\alpha}$ tensors and a common origin is employed, i.e. the shift $\boldsymbol{O}+\boldsymbol{a}$ is the same for all electrons, $\boldsymbol{O}_{l}+\boldsymbol{a}_{l}=\boldsymbol{O}_{1}+\boldsymbol{a}_{1}, \quad l=2, \ldots, N .{ }^{11}$ Alternatively, approaches such as Gauge Including Atomic Orbitals ${ }^{82,83}$ can be employed.

The velocity representation for the electronic part of the electric dipole moment can be 
derived via

$$
\begin{aligned}
\hbar\left(\omega_{k, e}-\omega_{i, 0}\right)\left\langle\psi_{i}^{(0)}\left|d_{\alpha}\right| \psi_{k}^{(e)}\right\rangle= & -e\left\langle\psi_{i}^{(0)}\left|\left[\sum_{l=1}^{N} r_{\alpha, l}, H^{(0)}\right]\right| \psi_{k}^{(e)}\right\rangle \\
= & -e\left\langle\psi_{i}^{(0)}\left|\frac{\mathrm{i} \hbar}{m_{e}} \sum_{l=1}^{N} p_{\alpha, l}\right| \psi_{k}^{(e)}\right\rangle \\
& -e\left\langle\psi_{i}^{(0)}\left|\left[\sum_{l=1}^{N} r_{\alpha, l}, V_{\text {nonloc }}\right]\right| \psi_{k}^{(e)}\right\rangle \\
= & \frac{\hbar}{\mathrm{i}}\left\langle\psi_{i}^{(0)}\left|\sum_{l=1}^{N} d_{\alpha, l}^{v}\right| \psi_{k}^{(e)}\right\rangle .
\end{aligned}
$$

This relation is exact in case of DFT calculations in the complete basis set limit. The second commutator arises if a non-local pseudopotential is used in the calculation. Additional terms may emerge in case of, e.g., spin-orbit coupling. ${ }^{73} \boldsymbol{v}=-\frac{\mathrm{i}}{\hbar}\left[\sum_{l=1}^{N} \boldsymbol{r}_{l}, H^{(0)}\right]$ is the velocity operator for the electrons and $\boldsymbol{d}^{\boldsymbol{v}}=-e \boldsymbol{v}=e \frac{\mathrm{i}}{\hbar}\left[\sum_{l=1}^{N} \boldsymbol{r}_{l}, H^{(0)}\right]$ the corresponding electric dipole operator for the electrons in the velocity representation.

The electric-dipole-electric-dipole tensor components in the velocity form are given by

$$
\alpha_{\alpha \beta}^{v}=f_{\text {occ }} \frac{2}{\hbar} \sum_{i, k} \frac{\left\langle\psi_{i}^{(0)}\left|d_{\alpha}^{v}\right| \psi_{k}^{(e)}\right\rangle\left\langle\psi_{k}^{(e)}\left|d_{\beta}^{v}\right| \psi_{i}^{(0)}\right\rangle}{\left(\omega_{k, e}-\omega_{i, 0}\right)^{3}}
$$

and, analogously, for the electric-dipole-magnetic-dipole tensor by

$$
G_{\alpha \beta}^{\prime s l, v}=-f_{\mathrm{occ}} \frac{2}{\hbar} \sum_{i, k} \frac{\left\langle\psi_{i}^{(0)}\left|d_{\alpha}^{v}\right| \psi_{k}^{(e)}\right\rangle\left\langle\psi_{k}^{(e)}\left|m_{\beta}\right| \psi_{i}^{(0)}\right\rangle}{\left(\omega_{k, e}-\omega_{i, 0}\right)^{3}} .
$$

$\boldsymbol{\alpha}^{\boldsymbol{v}}$ can be derived by first employing $d_{\beta}^{v}$, which leads, due to a vanishing first-order perturbed density, to an uncoupled set of linear equations and the KS orbitals $\left\{\left|\psi_{j, v}^{(1), \beta}\right\rangle\right\}$ :

$$
P \sum_{j}\left(H^{(0)} \delta_{i j}-\left\langle\psi_{i}^{(0)}\left|H^{(0)}\right| \psi_{j}^{(0)}\right\rangle\right) P\left|\psi_{j, v}^{(1), \beta}\right\rangle=P d_{\beta}^{v}\left|\psi_{i}^{(0)}\right\rangle .
$$

The KS orbitals $\left\{\left|\psi_{j, d_{v}}^{(0), \beta}\right\rangle\right\}=\mathrm{i}^{-1}\left\{\left|\psi_{j, v}^{(1), \beta}\right\rangle\right\}$ are used to obtain $\left\{\left|\psi_{j, d_{v}}^{(1), \beta}\right\rangle\right\}$ :

$$
\begin{aligned}
-P \sum_{j}\left(H^{(0)} \delta_{i j}-\left\langle\psi_{i}^{(0)}\left|H^{(0)}\right| \psi_{j}^{(0)}\right\rangle\right) P\left|\psi_{j, d_{v}}^{(1), \beta}\right\rangle & = \\
P & {\left[\left.\int \frac{\partial^{2} E_{\mathrm{Hxc}}[n(\boldsymbol{r})]}{\partial n(\boldsymbol{r}) \partial n\left(\boldsymbol{r}^{\prime}\right)}\right|_{n^{(0)}(\boldsymbol{r})} n_{d_{v}}^{(1), \beta}\left(\boldsymbol{r}^{\prime}\right) d \boldsymbol{r}^{\prime}\left|\psi_{i}^{(0)}\right\rangle+\left|\psi_{i, d_{v}}^{(0), \beta}\right\rangle\right] }
\end{aligned}
$$


with the first-order perturbed density

$$
n_{d_{v}}^{(1), \beta}(\boldsymbol{r})=\sum_{i} f_{\mathrm{occ}}\left[\psi_{i}^{(0) *}(\boldsymbol{r}) \psi_{i, d_{v}}^{(1), \beta}(\boldsymbol{r})+\psi_{i, d_{v}}^{(1), \beta *}(\boldsymbol{r}) \psi_{i}^{(0)}(\boldsymbol{r})\right]
$$

$\boldsymbol{\alpha}^{\boldsymbol{v}}$ and $\boldsymbol{G}^{\boldsymbol{s} \boldsymbol{s}, \boldsymbol{v}}$ are computed according to

$$
\begin{aligned}
\alpha_{\alpha \beta}^{v} & =-2 f_{\mathrm{occ}} \hbar^{2} \sum_{i}\left\langle\psi_{i, d_{v}}^{(1), \alpha} \mid \psi_{i, d_{v}}^{(0), \beta}\right\rangle, \\
G_{\alpha \beta}^{\prime s l, v} & =-2 f_{\mathrm{occ}} \hbar^{2} \operatorname{Im} \sum_{i}\left\langle\psi_{i, d_{v}}^{(1), \alpha} \mid \psi_{i, m}^{(1), \beta}\right\rangle .
\end{aligned}
$$

\section{Computational methodology}

The calculation of the ROA polarizability tensors has been implemented into the CP2K program package. $^{84}$ KS DFT was used as the electronic structure method with the Gaussian and plane waves method ${ }^{70}$ employing no periodic boundary conditions and Goedecker-Teter-Hutter (GTH) pseudopotentials. ${ }^{85-87}$ In all calculations, the BP86 exchangecorrelation density functional ${ }^{88,89}$ was chosen, which has been shown to provide reliable results for Raman and ROA spectra of biomolecules in the double-harmonic approximation. $^{20,21,90-94}$ Strict convergence criteria were applied in the calculations: The ground state orbitals were converged until the electronic energy was constant up to the eighth decimal place, and the conjugate gradient in the linear response calculation was converged up to the eleventh place. The kinetic energy cutoff for the plane wave expansion of the charge density was set to 280 Ry. A cubic simulation cell with a side length of $20 \AA$ containing one $S$-methyloxirane molecule was used in the calculations. For the calculations in the double-harmonic approximation, a step length of 0.01 bohr was employed for the calculation of the derivatives. The final spectra from the static calculations were obtained by applying a Lorentzian band width with a half-maximum height of $15 \mathrm{~cm}^{-1}$.

For the DFT-based MD calculation, the system has been held at around $20 \mathrm{~K}$ for 2 ps in the NVT ensemble using a canonical sampling through velocity rescaling thermostat ${ }^{95}$ 
followed by a production run in the NVE ensemble for 6 ps. The time step in the AIMD simulation was set to $0.3 \mathrm{fs}$, the ROA polarizability tensors were calculated for each time step. An extended version of the TRAVIS program ${ }^{50,96}$ was used to produce the spectrum applying zero padding and a Hanning type window function. Analogous to our

previous work, ${ }^{60}$ the harmonic approximation quantum correction factor $\frac{(\hbar \omega / k T)}{1-\exp (-\hbar \omega / k T)}(k$ : Boltzmann's constant, $T$ : temperature) was multiplied to the resulting ROA intensity differences.

\section{Example calculations}

As an example, backscattering ROA spectra for the $S$-methyloxirane molecule calculated in the double-harmonic approximation are shown in Fig. 1. In order to test the basis set dependence of these ROA calculations using GTH pseudopotentials and the Gaussian and plane waves approach, where wave functions are expanded in terms of a Gaussian basis set and the charge density is represented by means of an auxiliary plane wave basis, several calculations with different basis sets (see Fig. 1) as provided in the CP2K repository were performed. Going from a triple-zeta basis set with one set of polarization functions (TZVP-GTH) to a triple-zeta basis set with two sets of polarization functions (TZV2P-GTH) (see top of left-hand side in Fig. 1), several changes in ROA intensity differences are observed. The sign of the ROA intensity difference is not changed except of the band around $920 \mathrm{~cm}^{-1}$ corresponding to a normal mode with $\mathrm{C}-\mathrm{O}$ and exocyclic $\mathrm{C}-\mathrm{C}$ stretching vibrations. Calculation with the quadrupole-zeta basis set QZV2P-GTH leads to additional sign changes for the bands around $1080 \mathrm{~cm}^{-1}$ and $1240 \mathrm{~cm}^{-1}$, which belong to $\mathrm{CH}_{2}$ rocking and a mixture of ring $\mathrm{C}-\mathrm{C}$ stretching and bending motions of the hydrogen atom bound to the $\mathrm{C}^{*}$ center, respectively. No significant alterations are observed if the QZV3P-GTH basis set is used instead of the QZV2P-GTH basis set. Test calculations with the Gaussian 09 (G09) program, ${ }^{97}$ version revB.01, using BP86 and 
Ahlrich's def2-TZVP, def2-QZVP, def2-QZVPP'98,99 and the aug-cc-pVTZ and aug-ccpVQZ ${ }^{100}$ basis sets lead to a very similar result (see bottom of right-hand side of Fig. 1). Here, especially the bands around $1100 \mathrm{~cm}^{-1}$ show a remarkable basis set dependence as well (further studies about basis set dependence can be found in Refs. ${ }^{101,102}$ ).

Compared to the QZV3P-GTH result, no change of sign is obtained if several augmented GTH-basis sets are used (see top of right-hand side of Fig. 1). Using double- and triplezeta molopt basis sets ${ }^{103}$ (see bottom of left-hand side of Fig. 1), the most obvious basis set dependence leading to sign change of the ROA intensity differences is, similarly to the not augmented GTH basis sets mentioned above, detected for the bands around 930, 1100 , and $1250 \mathrm{~cm}^{-1}$. 
Figure 1: Backscattering ROA spectra of the $S$-methyloxirane molecule obtained within the double-harmonic approximation.
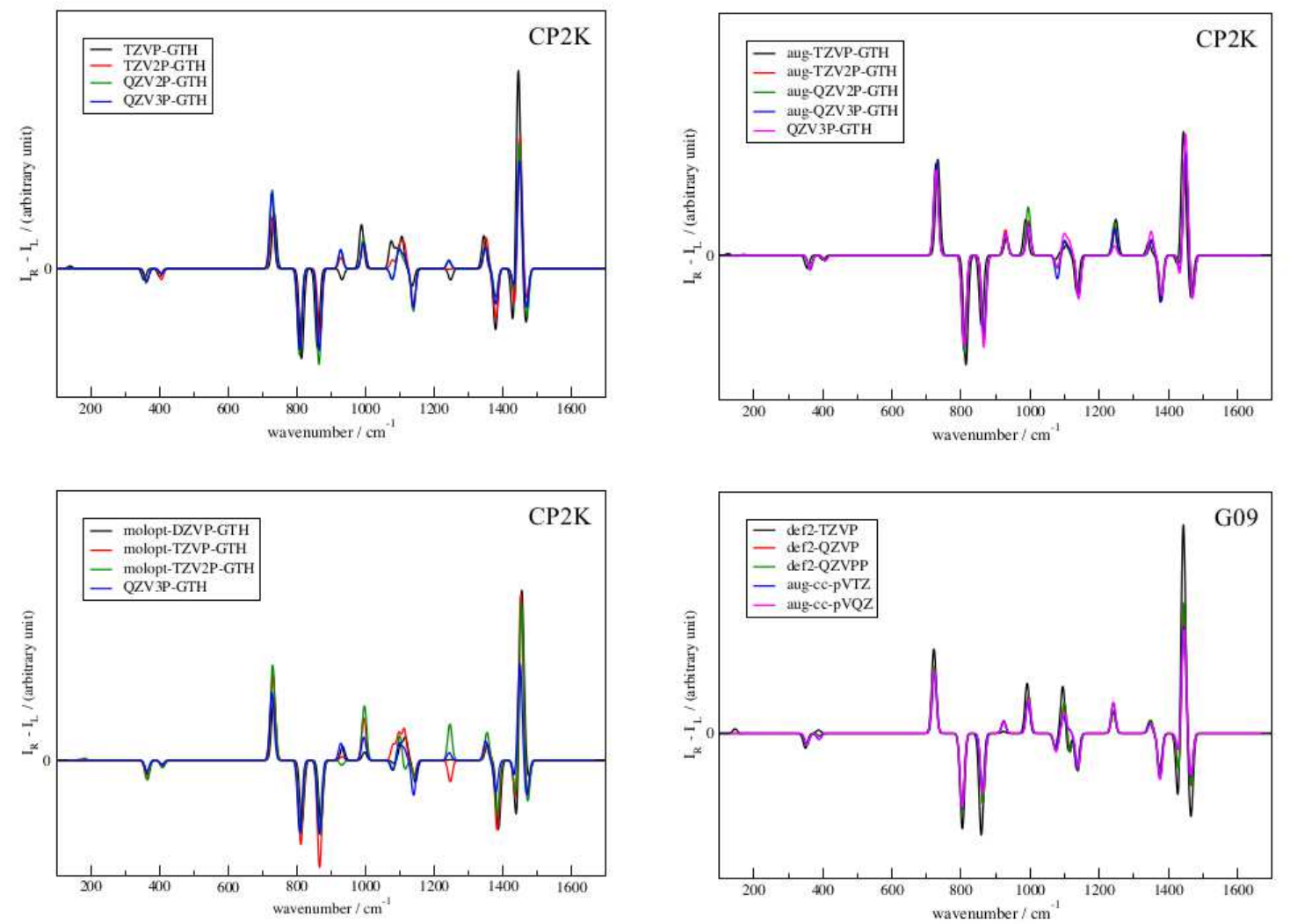

As shown in Eq. (21), it is in principle necessary to include the commutator $\left[\sum_{l=1}^{N} r_{\alpha, l}, V_{\text {nonloc }}\right]$ into the calculations if non-local pseudopotentials are used. Gonze and co-workers have investigated the influence of such commutator elements in calculations of optical matrix elements in a plane wave code. ${ }^{104}$ They found that the commutator contributions influence the peak heights of the electric-dipole-electric-dipole polarizability and that the size of correction is dependent on the method employed to generate the pseudopotentials. We have tested the effect of said matrix elements in the calculation of the ROA spectra of $S$-methyloxirane. As can be seen in Fig. 2, there are some amendments in the ROA 
intensity differences. Most noticeable is the considerably increased band height at about $1100 \mathrm{~cm}^{-1}$ as well as smaller intensity differences in the range from 1350 to $1500 \mathrm{~cm}^{-1}$ if the commutator is omitted. It can therefore not be concluded that the commutator contributions are always negligible and these matrix elements are thus included in the calculations.

Figure 2: Backscattering ROA spectra of the $S$-methyloxirane molecule obtained within the double-harmonic approximation using the TZVP-GTH basis set with and without consideration of the commutator given in Eq. (21).

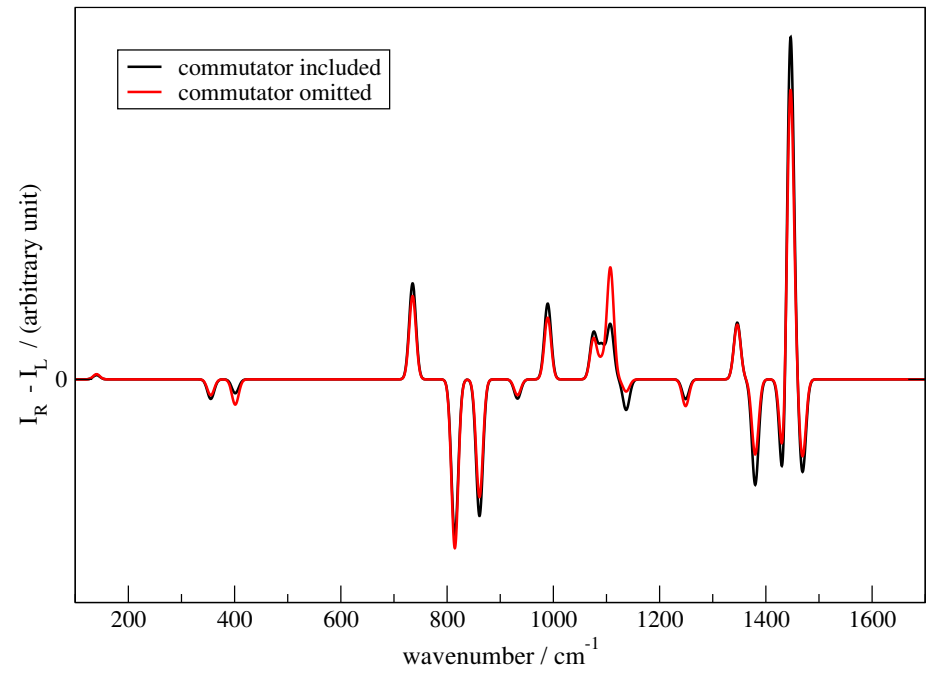

As an example for an ROA spectrum obtained from time correlation functions via DFTbased MD as described in Section 2, we have performed a DFT-based MD simulation for an $S$-methyloxirane molecule in gas phase (see Fig. 3) at a temperature of $20 \mathrm{~K}$. 
Figure 3: Backscattering ROA spectra of an $S$-methyloxirane molecule obtained from DFTbased MD using the QZV2P-GTH basis set and simulation lengths of 4, 5, and 6 ps.

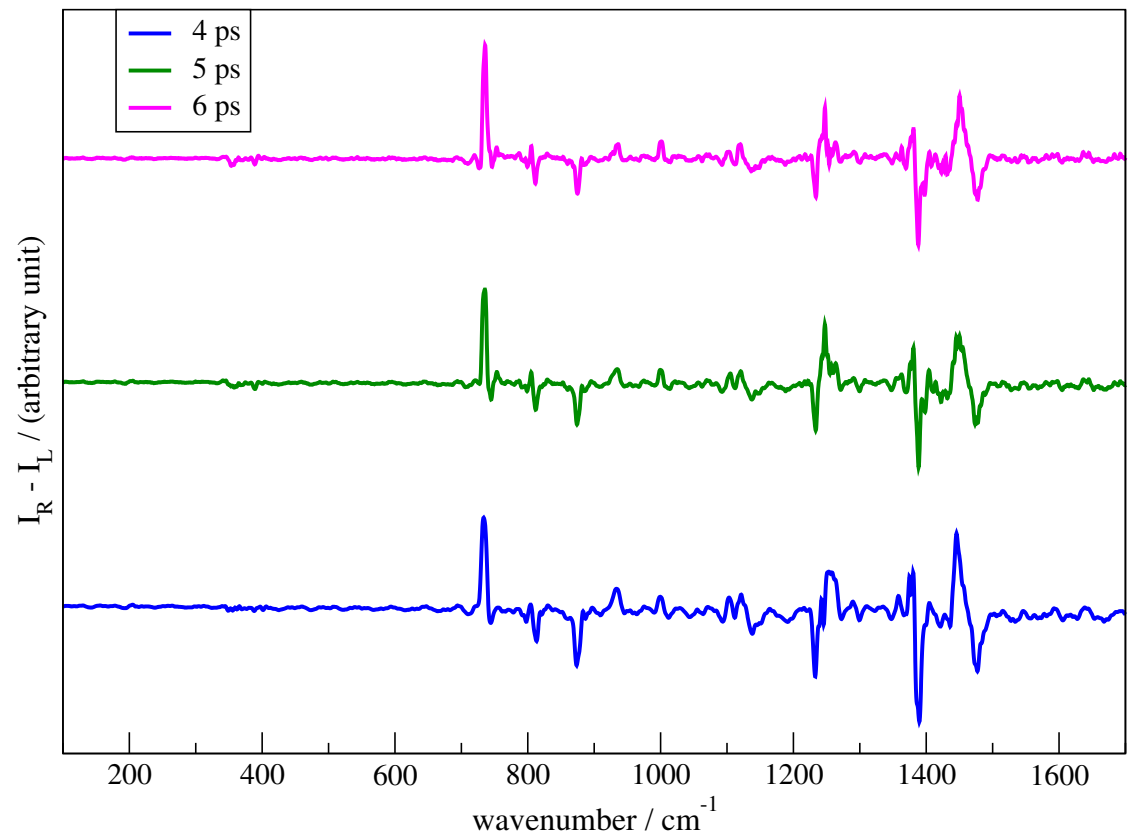

Due to the low temperature, temperature effects are less pronounced than in spectra calculated at room temperature, which facilitates the comparison to spectra obtained from the double-harmonic approximation assuming a temperature of $0 \mathrm{~K}$. Nevertheless, small barriers on the potential energy surface may still be overcome and anharmonicity is thus in principle included in the spectrum from DFT-based MD. ${ }^{105}$ Considering the short total simulation time of 6 ps and the low temperature applied, the calculated spectrum shows a reasonably good agreement with the calculated one in the double-harmonic approximation (see Fig. 1). All bands in the latter can be recognized in the DFT-based MD spectrum. Moreover, the signs of the ROA intensity differences agree well. The only remarkable exception is a negative band at around $1240 \mathrm{~cm}^{-1}$. Use of longer simulation times as well as generation of various microcanonical trajectories for calculation of an average 
ROA spectrum are therefore of interest for future studies in order to improve the quality of the ROA spectra computed by time correlation functions from DFT-based MD. It is worth mentioning that it is in general difficult in the calculation of gas-phase spectra from DFT-based MD to fulfill the equipartition theorem, which leads to deviations in the band intensities. ${ }^{48,50,105}$ Furthermore, DFT-based MD simulations at room temperature may be worth to be carried out as well as a more detailed study about the basis set and density functional dependence of the ROA DFT-based MD spectra in order to facilitate the comparison to experimental data. For condensed phase systems, taking into account periodic boundary conditions in the ROA calculations will be vital as well.

\section{$5 \quad$ Summary and Outlook}

We have described the calculation of ROA property tensors via computationally efficient DFPT, which has been implemented into the CP2K program using the Gaussian and plane waves method. Calculations relying on the double-harmonic approximation have been carried out where the gauge-origin independence has been ensured by utilizing the velocity representation for the electronic part of the electric dipole operator. Due to the non-local GTH pseudopotentials used, this requires the evaluation of additional terms compared to all-electron calculations, which do not necessarily lead to negligible contributions to the ROA intensity differences. Example calculations for $S$-methyloxirane in the double-harmonic approximation have shown that non-augmented GTH-basis sets of triple-zeta quality or less can lead to ROA bands featuring a wrong sign compared to results obtained close to the basis set limit. Moreover, a method for the calculation of ROA spectra from DFT-based MD has been developed based on time correlation functions, which naturally takes into account dynamic effects in contrast to the static approaches presented up to now. This paves the way for simulations of large systems at realistic thermodynamic conditions including anharmonicities and providing band shapes directly 
from the DFT-based MD simulation without the need for artificial broadening as required in static calculations. This approach has been applied for a DFT-based MD calculation of $S$-methyloxirane in the gas phase. Further investigations with respect to the influence of exchange-correlation density functional, basis set, and simulation lengths and temperature in the DFT-based MD approach may be worth to be carried out as well as the consideration of periodic boundary conditions for the calculation of condensed phase systems, which is especially of interest for complex biomolecules in solution.

\section{Acknowledgment}

Funding by Forschungskredit of the University of Zurich, grant no. FK-15-101, is gratefully acknowledged. The author thanks the Swiss National Supercomputing Center for computing resources (project ID: s502).

\section{References}

[1] Stephens, P. J. Theory of Vibrational Circular Dichroism J. Phys. Chem. 1985, 89, 748-752.

[2] Nakanishi, K.; Berova, N.; Woody, R. W. Circular Dichroism: Principles and Applications; Wiley-VCH: Weinheim, 2000.

[3] Atkins, P. W.; Barron, L. D. Rayleigh scattering of polarized photons by molecules Mol. Phys. 1969, 16, 453-466.

[4] Barron, L. D.; Buckingham, A. D. Rayleigh and Raman scattering from optically active molecules Mol. Phys. 1971, 20, 1111-1119. 
[5] Hug, W.; Kint, S.; Bailey, G.; Scherer, J. R. Raman circular intensity differential spectroscopy. Spectra of (-)-.alpha.-pinene and (+).alpha.-phenylethylamine $J$. Am. Chem. Soc. 1975, 97, 5589-5590.

[6] Barron, L. D.; Boogard, M. P.; Buckingham, A. D. Raman scattering of circularly polarized light by optically active molecules J. Am. Chem. Soc. 1973, 95, 603-605.

[7] Barron, L. D. The development of biomolecular Raman optical activity spectroscopy Biomed. Spectrosc. Imaging 2015, 4, 223-253.

[8] Kapitán, J.; Barron, L. D.; Hecht, L. A novel Raman optical activity instrument operating in the deep ultraviolet spectral region J. Raman Spectrosc. 2015, 46, 392-399.

[9] Šebestík, J.; Bouř, P. Observation of Paramagnetic Raman Optical Activity of Nitrogen Dioxide Angew. Chem. Int. Ed. 2014, 53, 9236-9239.

[10] Liégeois, V.; Ruud, K.; Champagne, B. An analytical derivative procedure for the calculation of vibrational Raman optical activity spectra J. Chem. Phys. 2007, 127, 204105.

[11] Luber, S.; Reiher, M. Raman optical activity spectra of chiral transition metal complexes Chem. Phys. 2008, 346, 212-223.

[12] Bouř, P.; Baumruk, V.; Hanzlikovà, J. Measurement and Calculation of the Raman Optical Activity of a-Pinene and trans-Pinane Collect. Czech. Chem. Commun. 1997, 62, 1384-1395.

[13] Jovan Jose, K. V.; Raghavachari, K. Raman Optical Activity Spectra for Large Molecules through Molecules-in-Molecules Fragment-Based Approach J. Chem. Theory Comput. 2016, 12, 585-594. 
[14] Crawford, T. D.; Ruud, K. Coupled-Cluster Calculations of Vibrational Raman Optical Activity Spectra ChemPhysChem 2011, 12, 3442-3448.

[15] Luber, S.; Neugebauer, J.; Reiher, M. Intensity-Tracking for Theoretical Infrared Spectroscopy of Large Molecules J. Chem. Phys. 2009, 130, 064105.

[16] Kiewisch, K.; Luber, S.; Neugebauer, J.; Reiher, M. Intensity Tracking for Vibrational Spectra of Large Molecules Chimia 2009, 63, 270-274.

[17] Luber, S.; Reiher, M. Intensity-carrying modes in Raman and Raman optical activity spectroscopy ChemPhysChem 2009, 10, 2049-2057.

[18] Pecul, M.; Rizzo, A.; Leszczynski, J. Vibrational Raman and Raman Optical Activity Spectra of $D$-Lactic Acid, $D$-Lactate, and $D$-Glyceraldehyde: Ab Initio Calculations J. Phys. Chem. A 2002, 106, 11008-11016.

[19] Cheeseman, J. R.; Shaik, M. S.; Popelier, P. L. A.; Blanch, E. W. Calculation of Raman Optical Activity Spectra of Methyl- $\beta$ - $D$-Glucose Incorporating a Full Molecular Dynamics Simulation of Hydration Effects J. Am. Chem. Soc. 2011, 133, 4991-4997.

[20] Jacob, C. R.; Luber, S.; Reiher, M. Understanding the signatures of secondarystructure elements in proteins via Raman optical activity spectroscopy Chem.-Eur. J. 2009, 15, 13491-13508.

[21] Luber, S.; Reiher, M. Theoretical Raman optical activity study of the $\beta$ domain of rat metallothionein J. Phys. Chem. B 2010, 114, 1057-1063.

[22] Kessler, J.; Kapitán, J.; Bouř, P. First-Principles Predictions of Vibrational Raman Optical Activity of Globular Proteins J. Phys. Chem. Lett. 2015, 6, 3314-3319.

[23] Weymuth, T.; Haag, M. P.; Kiewisch, K.; Luber, S.; Schenk, S.; Jacob, C. R.; Herrmann, C.; Neugebauer, J.; Reiher, M. MOVIPAC: Vibrational spectroscopy 
with a robust meta-program for massively parallel standard and inverse calculations J. Comput. Chem. 2012, 33, 1165-1175.

[24] Luber, S. Solvent Effects in Calculated Vibrational Raman Optical Activity Spectra of $\alpha$-Helices J. Phys. Chem. A 2013, 117, 2760-2770.

[25] Yamamoto, S. Conformational analyses of peptides and proteins by vibrational Raman optical activity Anal. Bioanal. Chem. 2012, 403, 2203-2212.

[26] Yamamoto, S.; Kaminsky, J.; Bouř, P. Structure and Vibrational Motion of Insulin from Raman Optical Activity Spectra Anal. Chem. 2012, 84, 2440-2451.

[27] Yamamoto, S.; Bouř, P. Transition Polarizability Model of Induced Resonance Raman Optical Activity J. Comput. Chem. 2013, 34, 2152-2158.

[28] Pecul, M.; Ruud, K. Ab Initio Calculation of Vibrational Raman Optical Activity Int. J. Quantum Chem. 2005, 104, 816-829.

[29] Crawford, T. D. Ab initio calculation of molecular chiroptical properties Theor. Chem. Acc. 2006, 115, 227-245.

[30] Ruud, K.; Thorvaldsen, A. J. Theoretical approaches to the calculation of Raman optical activity spectra Chirality 2009, 21, E54-E67.

[31] Lamparska, E.; Liégeois, V.; Quinet, O.; Champagne, B. Theoretical Determination of the Vibrational Raman Optical Activity Signatures of Helical Polypropylene Chains ChemPhysChem 2006, 7, 2366-2376.

[32] Drooghaag, J.; Marchand-Brynaert, J.; Champagne, B.; Liégeois, V. Combined Experimental and Theoretical Study on the Raman and Raman Optical Activity Signatures of Pentamethylundecane Diastereoisomers J. Phys. Chem. B 2010, 114, 11753-11760. 
[33] Jensen, L.; Autschbach, J.; Krykunov, M.; Schatz, G. C. Resonance vibrational Raman optical activity: A time-dependent density functional theory approach $J$. Chem. Phys. 2007, 127, 134101.

[34] Luber, S.; Neugebauer, J.; Reiher, M. Enhancement and De-enhancement Effects in Vibrational Resonance Raman Optical Activity J. Chem. Phys. 2010, 132, 044113.

[35] Luber, S.; Reiher, M. Prediction of Raman Optical Activity Spectra of Chiral 3-Acetylcamphorato-Cobalt Complexes ChemPhysChem 2010, 11, 1876-1887.

[36] Vidal, L. N.; Giovannini, T.; Cappelli, C. Can the Resonance Raman Optical Activity Spectrum Display Sign Alternation? J. Phys. Chem. Lett. 2016, 7, 35853590.

[37] Luber, S. Exploring Raman Optical Activity for Transition Metals: from Coordination Compounds to Solids Biomed. Spectrosc. Imaging 2015, 4, 255-268.

[38] Johannessen, C.; Hecht, L.; Merten, C. Comparative Study of Measured and Computed Raman Optical Activity of a Chiral Transition Metal Complex ChemPhysChem 2011, 12, 1419-1421.

[39] Humbert-Droz, M.; Oulevey, P.; Lawson Daku, L. M.; Luber, S.; Hagemann, H.; Bürgi, T. Where does the Raman optical activity of $\left[\mathrm{Rh}(\mathrm{en})_{3}\right]^{3+}$ come from? Insight from a combined experimental and theoretical approach Phys. Chem. Chem. Phys. 2014, 16, 23260-23273.

[40] Hug, W. Visualizing Raman and Raman optical activity generation in polyatomic molecules Chem. Phys. 2001, 264, 53-69.

[41] Fedorovsky, M. Exploring Vibrational Optical Activity with PyVib2 Comput. Lett. 2006, 2, 233-236. 
[42] Liégeois, V.; Champagne, B. Implementation in the Pyvib2 program of the localized mode method and application to a helicene Theor. Chem. Acc. 2012, 131, 1284.

[43] Ramírez, R.; López-Ciudad, T.; Kumar P, P.; Marx, D. Quantum corrections to classical time-correlation functions: Hydrogen bonding and anharmonic floppy modes J. Chem. Phys. 2004, 121, 3973.

[44] Roy, T. K.; Gerber, R. B. Vibrational self-consistent field calculations for spectroscopy of biological molecules: new algorithmic developments and applications Phys. Chem. Chem. Phys. 2013, 15, 9468-9492.

[45] Daněček, P.; Kapitán, J.; Baumruk, V.; Bednárová, L.; V. Kopecký, J.; Bouř, P. Anharmonic effects in IR, Raman, and Raman optical activity spectra of alanine and proline zwitterions J. Chem. Phys. 2007, 126, 224513.

[46] Bloino, J.; Biczysko, M.; Barone, V. Anharmonic Effects on Vibrational Spectra Intensities: Infrared, Raman, Vibrational Circular Dichroism, and Raman Optical Activity J. Phys. Chem. A 2015, 119, 11862-11874.

[47] Panek, P. W.; Jacob, C. R. Anharmonic Theoretical Vibrational Spectroscopy of Polypeptides J. Phys. Chem. Lett. 2016, 7, 3084-3090.

[48] Gaigeot, M.-P.; Spezia, R. Theoretical Methods for Vibrational Spectroscopy and Collision Induced Dissociation in the Gas Phase Top. Curr. Chem. 2015, 364, 99151.

[49] Gaigeot, M.-P.; Martinez, M.; Vuilleumier, R. Infrared spectroscopy in the gas and liquid phase from first principle molecular dynamics simulations: application to small peptides Mol. Phys. 2007, 105, 2857-2878.

[50] Thomas, M.; Brehm, M.; Fligg, R.; Vöhringer, P.; Kirchner, B. Computing vibrational spectra from ab initio molecular dynamics Phys. Chem. Chem. Phys. 2013, 15, 6608-6622. 
[51] Sulpizi, M.; Salanne, M.; Sprik, M.; Gaigeot, M.-P. Vibrational sum frequency generation spectroscopy of the water liquid-vapor interface from density functional theory-based molecular dynamics simulations J. Phys. Chem. Lett. 2013, 4, 83-87.

[52] Ohto, T.; Usui, K.; Hasegawa, T.; Bonn, M.; Nagata, Y. Toward ab initio molecular dynamics modeling for sum-frequency generation spectra; an efficient algorithm based on surface-specific velocity-velocity correlation function J. Chem. Phys. 2015, 143, 124702.

[53] Ohto, T.; Backus, E. H. G.; Hsieh, C.-S.; Sulpizi, M.; Bonn, M.; Nagata, Y. Lipid carbonyl groups terminate the hydrogen bond network of membrane-bound water J. Phys. Chem. Lett. 2015, 6, 4499-4503.

[54] Wan, Q.; Galli, G. First-Principles Framework to Compute Sum-Frequency Generation Vibrational Spectra of Semiconductors and Insulators Phys. Rev. Lett. 2015, 115, 246404.

[55] Ohto, T.; Backus, E. H. G.; Mizukami, W.; Hunger, J.; Bonn, M.; Nagata, Y. Unveiling the amphiphilic nature of tmao by vibrational sum frequency generation spectroscopy J. Phys. Chem. C 2016, 120, 17435-17443.

[56] Luber, S. Sum frequency generation of acetonitrile on rutile (110) surface from density functional theory-based molecular dynamics J. Phys. Chem. Lett. 2016, 7, 5183-5187.

[57] Putrino, A.; Parrinello, M. Anharmonic Raman Spectra in High-Pressure Ice from Ab Initio Simulations Phys. Rev. Lett. 2002, 88, 176401.

[58] Pagliai, M.; Cavazzoni, C.; Cardini, G.; Erbacci, G.; Parrinello, M.; Schettino, V. Anharmonic infrared and Raman spectra in Car-Parrinello molecular dynamics simulations J. Chem. Phys. 2008, 128, 224514. 
[59] Wan, Q.; Spanu, L.; Galli, G. A.; Gygi, F. Raman Spectra of Liquid Water from Ab Initio Molecular Dynamics: Vibrational Signatures of Charge Fluctuations in the Hydrogen Bond Network J. Chem. Theory Comput. 2013, 9, 4124-4130.

[60] Luber, S.; Iannuzzi, M.; Hutter, J. Raman spectra from ab initio molecular dynamics and its application to liquid S-methyloxirane J. Chem. Phys. 2014, 141, 094503.

[61] Thomas, M.; Kirchner, B. Classical Magnetic Dipole Moments for the Simulation of Vibrational Circular Dichroism by ab Initio Molecular Dynamics J. Phys. Chem. Lett. 2016, 7, 509-513 ; after submission of this manuscript, another VCD study using DFT-based MD has been published: A. Scherrer, R. Vuilleumier, D. Sebastiani, DOI: http://dx.doi.org/10.1063/1.4960653.

[62] Hopmann, K. H.; Ruud, K.; Pecul, M.; Kudelski, A.; Dračinský, M.; Bouř, P. Explicit versus Implicit Solvent Modeling of Raman Optical Activity Spectra $J$. Phys. Chem. B 2011, 115, 4128-4137.

[63] Kaminski, M.; Kudelski, A.; Pecul, M. Vibrational Optical Activity of Cysteine in Aqueous Solution: A Comparison of Theoretical and Experimental Spectra $J$. Phys. Chem. B 2012, 116, 4976-4990.

[64] Urago, H.; Suga, T.; Hirata, T.; Kodama, H.; Unno, M. Raman Optical Activity of a Cyclic Dipeptide Analyzed by Quantum Chemical Calculations Combined with Molecular Dynamics Simulations J. Phys. Chem. B 2014, 118, 6767-6774.

[65] Zielinski, F.; Mutter, S. T.; Johannessen, C.; Blanch, E. W.; Popelier, P. L. A. The Raman optical activity of $\beta$ - $D$-xylose: where experiment and computation meet Phys. Chem. Chem. Phys. 2015, 17, 21799-21809. 
[66] Melcová, A.; Kessler, J.; Bouř, P.; Kaminský, J. Simulation of Raman optical activity of multi-component monosaccharide samples Phys. Chem. Chem. Phys. 2016, 18, 2130-2142.

[67] Mutter, S. T.; Zielinski, F.; Johannessen, C.; Popelier, P. L. A.; Blanch, E. W. Distinguishing Epimers Through Raman Optical Activity J. Phys. Chem. A 2016, 120, 1908-1916.

[68] Oulevey, P.; Luber, S.; Varnholt, B.; Bürgi, T. Symmetry Breaking in Chiral Ionic Liquids Evidenced by Vibrational Optical Activity Angew. Chem. Int. Ed. 2016, $55,11787-11790$.

[69] Horniček, J.; Kaprálová, P.; Bouř, P. Simulations of vibrational spectra from classical trajectories: Calibration with ab initio force fields J. Chem. Phys. 2007, $127,084502$.

[70] VandeVondele, J.; Krack, M.; Mohamed, F.; Parrinello, M.; Chassaing, T.; Hutter, J. Quickstep: Fast and accurate density functional calculations using a mixed Gaussian and plane waves approach Comput. Phys. Commun. 2005, 167, 103-128.

[71] Wilson, Jr., E. B.; Decius, J. C.; Cross, P. C. Molecular Vibrations; McGraw-Hill: New York, 1955.

[72] Long, D. A. The Raman Effect: A Unified Treatment of the Theory of Raman Scattering by Molecules; John Wiley \& Sons: New York, 2002.

[73] Barron, L. D. Molecular Light Scattering and Optical Activity; Cambridge University Press: Cambridge, 2nd ed.; 2004.

[74] Placzek, G. Rayleigh-Streuung und Raman-Effekt. In Handbuch der Radiologie, Vol. 6; Marx, E., Ed.; Akademische Verlagsgesellschaft: Leipzig, 1934. 
[75] Black, T. M.; Bose, P. K.; Polavarapu, P. L.; Barron, L. D.; Hecht, L. Vibrational Activity in trans-2,3-Dimethyloxirane J. Am. Chem. Soc. 1990, 112, 1479-1489.

[76] Amos, R. D. Electric And Magnetic Properties Of CO, HF, $\mathrm{HCl}$ and $\mathrm{CH}_{3} \mathrm{~F}$ Chem. Phys. Lett. 1982, 87, 23-26.

[77] Gonze, X. Adiabatic density-functional perturbation theory Phys. Rev. A 1995, 52, 1096-1114.

[78] Gonze, X.; Vigneron, J.-P. Density-functional approach to nonlinear-response coefficients of solids Phys. Rev. B 1989, 39, 13120-13128.

[79] Putrino, A.; Sebastiani, D.; Parrinello, M. Generalized variational density functional perturbation theory J. Chem. Phys. 2000, 113, 7102.

[80] Coriani, S.; Pecul, M.; Rizzo, A.; Jørgensen, P.; Jaszuński, M. Ab initio study of magnetochiral birefringence J. Chem. Phys. 2006, 117, 6417-6428.

[81] Buckingham, A. D.; Longuet-Higgins, H. C. The quadrupole moments of dipolar molecules Mol. Phys. 1968, 14, 63-72.

[82] London, F. Quantum theory of interatomic currents in aromatic compounds J. Phys. Radium 1937, 8, 397-409.

[83] Helgaker, T.; Ruud, K.; Bak, K. L.; Jørgensen, P.; Olsen, J. Vibrational Raman Optical Activity Calculations using London Atomic Orbitals Faraday Discuss. 1994, 99, 165-180.

[84] CP2K Developers Group, URL: http://www.cp2k.org.

[85] Goedecker, S.; Teter, M.; Hutter, J. Separable dual-space Gaussian pseudopotentials Phys. Rev. B 1996, 54, 1703-1710. 
[86] Hartwigsen, C.; Goedecker, S.; Hutter, J. Relativistic separable dual-space Gaussian pseudopotentials from H to Rn Phys. Rev. B 1998, 58, 3641-3662.

[87] Krack, M. Pseudopotentials for H to Kr optimized for gradient-corrected exchangecorrelation functionals Theor. Chem. Acc. 2005, 114, 145-152.

[88] Becke, A. D. Density-functional exchange-energy approximation with correct asymptotic behavior Phys. Rev. A 1988, 38, 3098-3100.

[89] Perdew, J. P. Density-functional approximation for the correlation energy of the inhomogeneous electron gas Phys. Rev. B 1986, 33, 8822-8824.

[90] Luber, S.; Herrmann, C.; Reiher, M. Relevance of the Electric-Dipole-ElectricQuadrupole Contribution to Raman Optical Activity Spectra J. Phys. Chem. B 2008, 112, 2218-2232.

[91] Jacob, C. R.; Luber, S.; Reiher, M. Calculated Raman Optical Activity Signatures of Tryptophan Side Chains ChemPhysChem 2008, 9, 2177-2180.

[92] Jacob, C. R.; Luber, S.; Reiher, M. Analysis of secondary structure effects on the IR and Raman spectra of polypeptides in terms of localized vibrations J. Phys. Chem. B 2009, 113, 6558-6573.

[93] Luber, S.; Reiher, M. Calculated Raman optical activity spectra of 1,6-Anhydro$\beta$-D-glucopyranose J. Phys. Chem. A 2009, 113, 8268-8277.

[94] Varnholt, B.; Oulevey, P.; Luber, S.; Kumara, C.; Dass, A.; Bürgi, T. Structural Information on the Au-S Interface of Thiolate-Protected Gold Clusters: A Raman Spectroscopy Study J. Phys. Chem. C 2014, 118, 9604-9611.

[95] Bussi, G.; Donadio, D.; Parrinello, M. Canonical sampling through velocity rescaling J. Chem. Phys. 2007, 126, 014101. 
[96] Brehm, M.; Kirchner, B. TRAVIS - A Free Analyzer and Visualizer for Monte Carlo and Molecular Dynamics Trajectories J. Chem. Inf. Model. 2011, 51, 2007-2023.

[97] Frisch, M. J. et al. "Gaussian 09 Revision B.01", Gaussian Inc. Wallingford CT 2009.

[98] Weigend, F.; Ahlrichs, R. RI-MP2: optimized auxiliary basis sets and demonstration of efficiency Phys. Chem. Chem. Phys. 1998, 294, 143-152.

[99] Weigend, F.; Furche, F.; Ahlrichs, R. Gaussian basis sets of quadruple zeta valence quality for atoms H-Kr J. Chem. Phys. 2003, 119, 12753-12762.

[100] Kendall, R. A.; Dunning, T. H.; Harrison, R. J. Electron affinities of the first-row atoms revisited. Systematic basis sets and wave functions J. Chem. Phys. 1992, 96, 6796-6806.

[101] Cheeseman, J. R.; Frisch, M. J. Basis Set Dependence of Vibrational Raman and Raman Optical Activity Intensities J. Chem. Theory Comput. 2011, 7, 3323-3334.

[102] Reiher, M.; Liégeois, V.; Ruud, K. Basis Set and Density Functional Dependence of Vibrational Raman Optical Activity calculations J. Phys. Chem. A 2005, 109, 7567-7574.

[103] VandeVondele, J.; Hutter, J. Gaussian basis sets for accurate calculations on molecular systems in gas and condensed phases J. Chem. Phys. 2007, 127, 114105.

[104] Motta, C.; Giantomassi, M.; Cazzaniga, M.; Gaál-Nagy, K.; Gonze, X. Implementation of techniques for computing optical properties in 0-3 dimensions, including a real-space cutoff, in ABINIT Comput. Mater. Sci. 2010, 50, 698-703.

[105] Cimas, A.; Maitre, P.; Ohanessian, G.; Gaigeot, M.-P. Molecular Dynamics and Room Temperature Vibrational Properties of Deprotonated Phosphorylated Serine J. Chem. Theory Comput. 2009, 5, 2388-2400. 


\section{for Table of Contents use only}

"Raman optical activity spectra from density functional perturbation theory and density functional theory-based molecular dynamics" by Sandra Luber

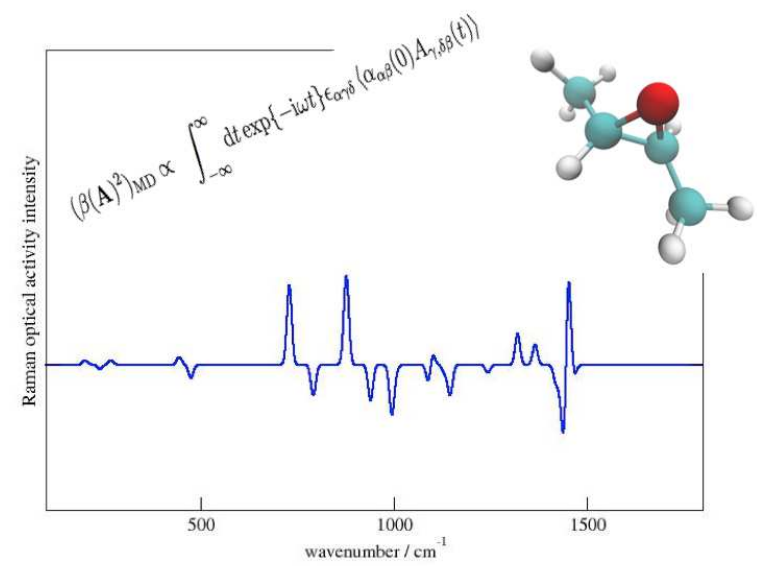

